\title{
A Transmissive Optics Approach for Time-Slicing the LCLS X-Ray Pulse
}

\author{
R.M. Bionta
}

May 17,2000

U.S. Department of Energy

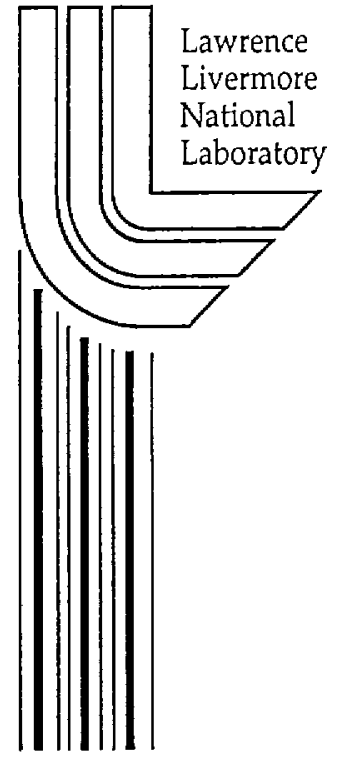




\section{DISCLAIMER}

This document was prepared as an account of work sponsored by an agency of the United States Government. Neither the United States Government nor the University of California nor any of their employees, makes any warranty, express or implied, or assumes any legal liability or responsibility for the accuracy, completeness, or usefulness of any information, apparatus, product, or process disclosed, or represents that its use would not infringe privately owned rights. Reference herein to any specific commercial products, process, or service by trade name, trademark, manufacturer, or otherwise, does not necessarily constitute or imply its endorsement, recommendation, or favoring by the United States Government or the University of California. The views and opinions of authors expressed herein do not necessarily state or reflect those of the United States Government or the University of California, and shall not be used for advertising or product endorsement purposes.

This report has been reproduced directly from the best available copy. '

Available to DOE and DOE contractors from the Office of Scientific and Technical Information

P.O. Box 62, Oak Ridge, TN 37831

Prices available from (615) 576-8401, FTS 626-8401

Available to the public from the National Technical Information Service

U.S. Department of Commerce

5285 Port Royal Rd.,

Springfield, VA 22161 


\section{BEST AVAILABLE COPY}

FOR ORIGINAL RISPORT

CALL

REPORTS LIBRARY

$\times 37097$ 
Physics Department, N

\section{Division}

Lawrence Livermore

National Laboratory

\section{A transmissive optics approach for time-slicing the LCLS x-ray pulse.}

R. M. Bionta

May 17,2000

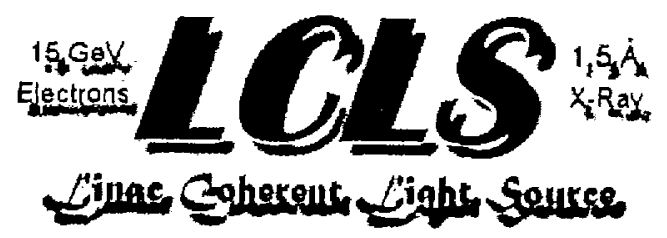




\title{
A transmissive optics approach for time- slicing the LCLS $x$-ray pulse.
}

\begin{abstract}
This paper investigates the use of off-axis zone plate optical systems to deliver time-sliced LCLS FEL pulses to users under the 3 energy chirp scenarios elucidated by P. Emma. We present formulas for designing off-axis zone plate optical systems that achieve a given time-slice duration and intensity. The results show that it is feasible to fabricate zone-plate systems capable of providing intense spots of time-sliced $8.275 \mathrm{KeV}$ photons under the scenario of a $2.0 \%$ chirp, but that it is beyond current and envisioned fabrication capabilities to create zone-plate systems of similar performance under the scenarios offering energy chirps of $<0.25 \%$. Finally we present results of numerical calculations of the electric fields delivered to the user by an off-axis zone plate optical system producing time-slices of $\leq 50 \cdot f s$ with photon densities of 200 photons $/ \AA^{2}$ under the $2 \%$ energy chirp scenario.
\end{abstract}

\section{Introduction}

There is much interest in schemes to obtain $x$-ray pulses of shorter duration than the nominal 255 fs LCLS FEL pulse train. For example, research by Janos Hajdu and his colleagues have shown the utility of intense $x$-ray pulses of less than $50 \mathrm{fs}$ duration for imaging single biological molecules ${ }^{1}$. A short pulse overcomes the problem that the number of photons required to obtain structural information from a single molecule is far above the threshold for breaking apart the molecule. This threshold for molecular breakup occurs at a photon density of 200 photons $/ \AA^{2}$. The calculations by Hajdu's group indicate that by delivering all of these photons in less than 50 fs the molecule does not have time to break up. The LCLS Scientific Advisory Committee has identified this topic in its list of five "priority" experiments.

There are two fundamental approaches for obtaining shorter LCLS pulses both based on manipulating the electrons delivered to the undulator. The first approach is to attempt to shorten the length of the delivered electron bunch, directly resulting in a shorter duration FEL pulse. The second approach is to deliver an electron bunch that has an energy "chirp" along its length which results in an FEL pulse of nominal duration but whose fundamental wavelength changes with time from the beginning of the pulse to the end of the pulse. Presumably the chiped $x$-ray pulse may be manipulated optically to provide pulses of shorter duration. The optical methods for deriving shorter duration pulses may be divided into pulse-compression techniques, which attempt to compress the pulse in time while presenving its total energy, and time-slicing techniques, which attempt to extract a short segment of the longer pulse.

This paper presents detailed calculations of a time-slicing technique based on off-axis transmissive zone plates. Although, in principle, the pulse-compression techniques promise higher intensities than time-slicing techniques, quantitative estimates of the intensity and duration that can be achieved by proposed pulse-compression techniques are difficult and require detailed consideration of optical efficiencies, power loading, and delivery of the compressed pulse to the user. The simplicity of the time-slicing technique presented here allow detailed calculations of the pulse shape and intensity delivered to the user and should serve as a starting point for what is practical at the LCLS. 


\section{Possibilities for chirping the electron bunch as elucidated by P. Emma}

Possible scenarios for generating a linear time-correlated energy chirp in the electron bunch, prior to insertion in the undulator were explored in the paper b1y P. Emma ${ }^{3}$. These scenarios are summarized in the following table which lists the nominal LCLS configuration as well as 4 possible cases:

\begin{tabular}{|c|c|c|c|c|c|}
\hline $\begin{array}{l}\text { compression } \\
\text { scenario }\end{array}$ & $\begin{array}{c}e^{-} F W H M \\
\text { bunch length } \\
T \\
\text { [fsec] }\end{array}$ & $\begin{array}{c}e^{-} \text {FWHM } \\
\text { energy } \\
\text { spread } \\
\\
{[\%]}\end{array}$ & $\begin{array}{c}y \text { energy } \\
\text { increase at } \\
\text { beginning of } \\
\text { pulse } \\
8.271 \mathrm{KeV} \\
\Delta \\
{[\%]}\end{array}$ & Charge & $\begin{array}{c}\text { CSR / } \\
\text { wakefield } \\
\text { 'risk' }\end{array}$ \\
\hline $\begin{array}{c}\text { Nominal } \\
\text { case-1 } \\
\text { case-2 } \\
\text { case-3 } \\
\text { case-4 }\end{array}$ & $\begin{array}{c}255 \\
10 \\
130 \\
670 \\
255\end{array}$ & $\begin{array}{c}0.06 \\
0.07 \\
+0.20 \\
+0.25 \\
+2.00\end{array}$ & $\begin{array}{l}+0.20 \\
-0.25 \\
+2.00\end{array}$ & $\begin{array}{c}1 \\
0.2 \\
1 \\
1 \\
1\end{array}$ & $\begin{array}{c}\text { nominal } \\
\text { high } \\
\text { high } \\
\text { low } \\
\text { high }\end{array}$ \\
\hline
\end{tabular}

- Table 1 - Electron bunch compression and energy chipp scenarios.

For each case the table gives the nominal bunch length, $T$, the \% spread in the electron kinetic energy from the beginning to the end of the pulse, the net increase (or decrease) in the FEL photon energy above nominal at the beginning of the pulse, $\Delta$, the expected bunch charge, and in the last column Emma's estimate of the risk that coherent synchrotron radiation emission in each configuration will destroy the conditions necessary for lasing.

Case 1 is a direct attempt to shorten the FEL pulse by shortening the electron bunch and will not be further discussed here. Case 2 results in a 130 fs long pulse with a small photon energy chip of $\pm 0.2 \%$. Case 3 results in a long 670 fs pulse also with a small \pm $0.25 \%$ photon energy chirp. Case 4 results in a nominal pulse length of 255 fs but with an impressive $\pm 2.0 \%$ photon energy spread. Clearly case 4 will give the best performance in any optical time-slicing scheme but only case 3 , which will give the poorest performance, is listed as low risk. Nevertheless, case 3 is the only scenario where the lower energy photons are at the beginning of the pulse, a possible advantage for optical pulse compression, which will not be discussed here.

\section{Off-axis zone plate time slice technique}

The following discusses how an off-axis zone plate in combination with a slit can be used to select a finite slice of the energy chirped photon beam resulting in a pulse of shorter duration.

The basic idea of this technique, shown schematically in figure 1 , is that a circular zone plate focuses light to a point on its optical axis whose distance from the zone plate is a function of wavelength. If the illumination is restricted to a small area near the outer edge of the zone plate, it will still converge to the focal point on the zone plate axis, giving the appearance that the small beam has been deflected sideways as if by a grating (but unlike a grating, the light is also focused). In the case of a chiped FEL pulse, where the wavelength decreases monotonically over the duration of the pulse, the focal spot will move outward along the zone plate axis. An observer looking at a screen placed 


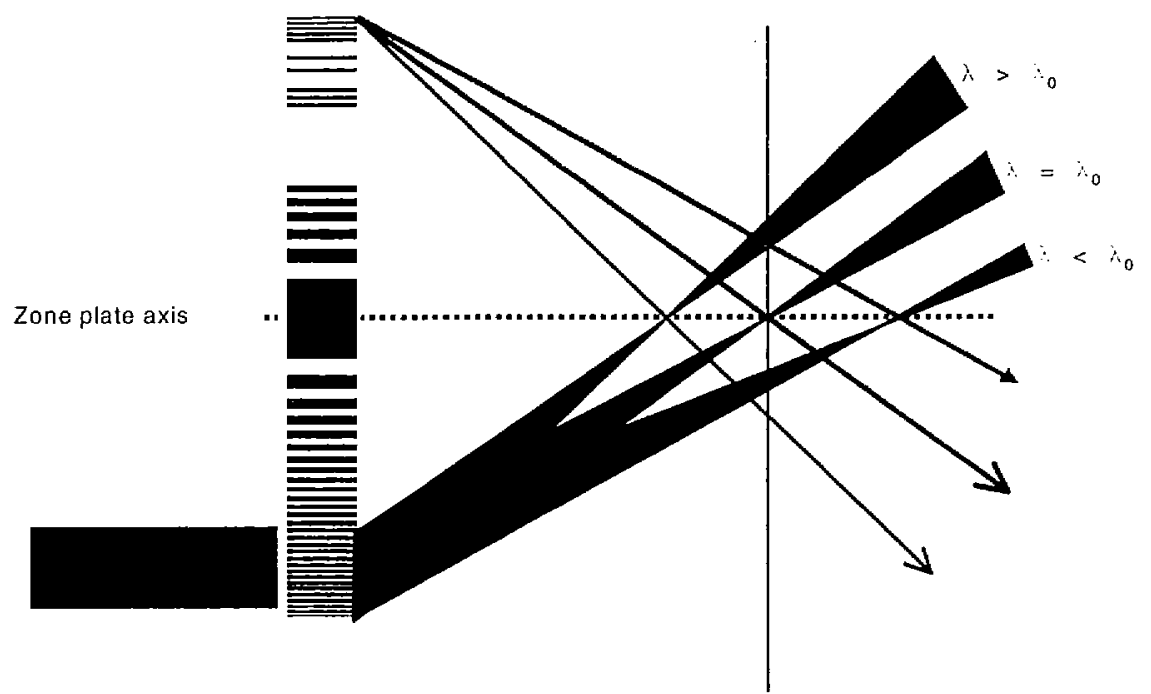

- Figure 1 - Image formation by zone plate struck off axis at different wavelengths. During a wavelength chirpped FEL pulse the focused spot will move along the zone-plate axis causing the beam to sweep across the plane of the central focus.

perpendicular to the zone plate axis at the distance of the mid-pulse focus will see a small spot of light moving across the screen during the pulse. Placing a small pinhole through the screen in the path of the spot produces a brief pulse of light downstream whose duration is roughly equal to the original pulse duration times the ratio of the width of the pinhole to the total path length of the moving spot. The concept also works with off-axis linear zone plates, which produce a moving line of light of lower power density. Note that the entire zone plate does not have to be manufactured, only the portion intercepted by the FEL beam. For large offsets, the required off-axis zone plate portion closely resembles a grating having zones of nearly equal thickness across its aperture.

\section{Sputtered-sliced transmissive zone plates for LCLS}

Both linear and circular transmissive zone plates and linear gratings, operating at $8 \mathrm{KeV}$, have been manufactured using the sputter-slice technique. ${ }^{4}$ In this technique, see figure 2 , linear structures are made by sputtering alternating layers of two materials onto a flat substrate and slicing the resulting structure perpendicular to the substrate surface. A uniform $x$-ray wave front traveling through the slice in a direction parallel to the layers emerges from the other side of the sliced optic with an alternating pattem of amplitude and phase modulation produced by the differing optical constants of the two materials making up the layers. Interference between the x-rays emerging from the two different materials produces the well-known diffraction pattems associated with diffraction gratings and zone plates of everyday visible optics. Circular zone plates have also been made with this technique by sputtering the layers onto a wire core.

The chief advantage of this technique is that zone plates and gratings with large aspect ratios can be produced, where aspect ratio refers to the thickness of the optic along the $x$ ray beam direction (in this case the slice thickness) to the width of the zones (in this case the depth of the sputtered layers.) Large aspect ratios are necessary to give sufficient optical power at high $x$-ray energies and have the added advantage at the LCLS that they allow the use of lower $Z$ materials, which tend to suffer lower doses in the FEL beam.

The chief disadvantage of the sputtered-sliced technique is that it is difficult to sputter very thick multilayer deposits without inducing columnar defects that spoil the upper layers. 
This places practical upper limits on the aperture of the lens and lower limits on the minimum zone size. These limits are strongly materials dependent. In general, sputteredsliced lenses produced to date have apertures in the range of 10 to $250 \mu \mathrm{m}$ with minimum feature sizes in the range of 0.07 to $0.4 \mu \mathrm{m}$.
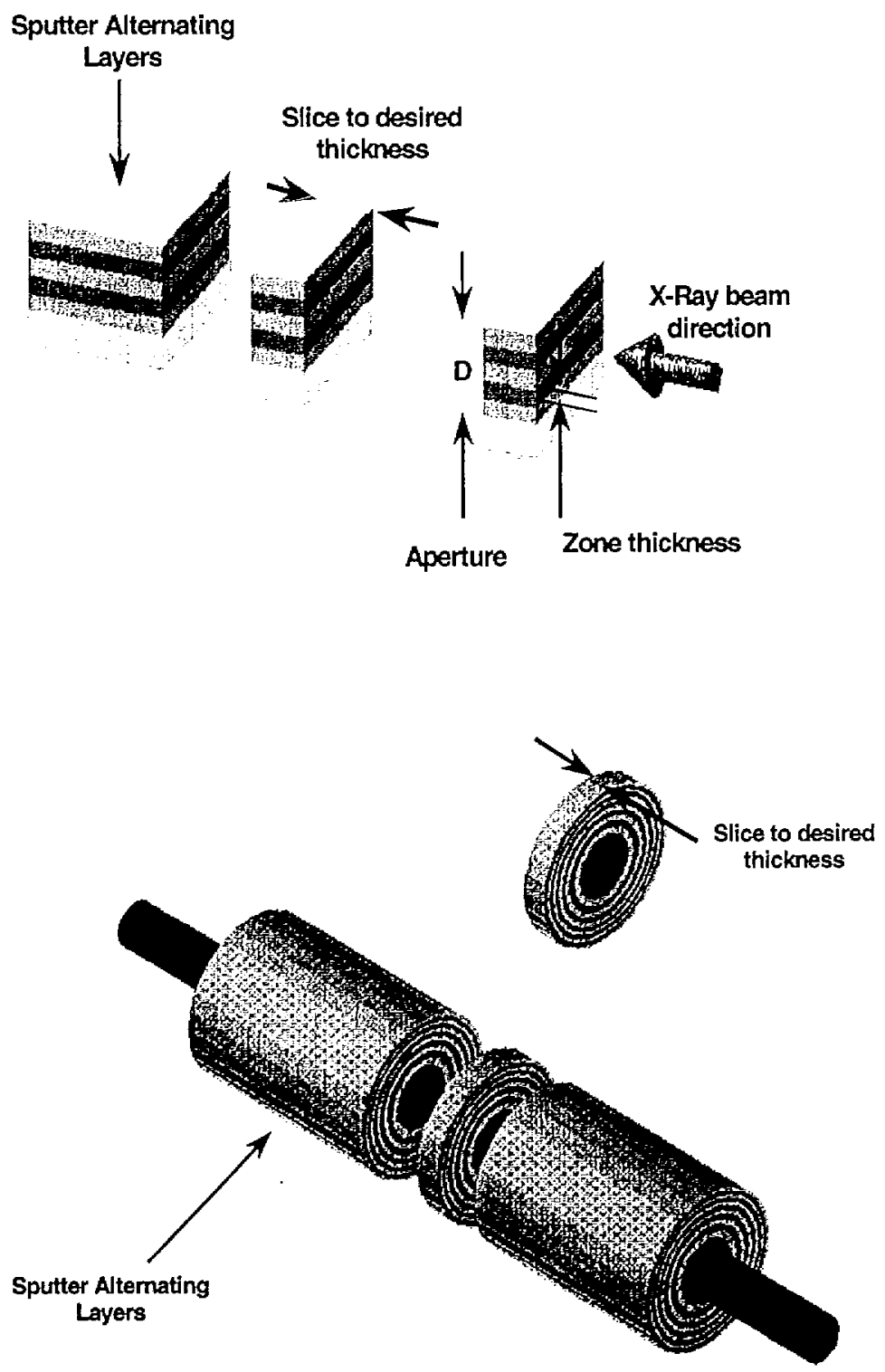

- Figure 2 - Sputter-sliced method for fabricating transmissive optics with high aspect ratio. Top, fabrication of linear gratings and zone plates. Bottom, fabrication of circular zone plates. 


\section{Materials for transmissive optics at LCLS}

Both amplitude and phase modulating sputtered-sliced devices have been reported in the cited literature operating with $8 \mathrm{KeV} \times$ rays. Amplitude modulating devices were made of aluminum and tantalum sliced to thickness of $17 \mu \mathrm{m}$, a thickness where at $8 \mathrm{KeV}$ the aluminum is fairly transparent while the tantalum is essentially opaque. The phase modulating devices were made of aluminum and copper sliced to a thickness of $7 \mu \mathrm{m}$. At a thickness of $7 \mu \mathrm{m}$, aluminum and copper are fairly transparent to $8 \mathrm{KeV} \times$ rays, but interference is caused by the $1-\pi$ relative phase shift imparted by the two materials. Unfortunately all of these materials will suffer from high doses at the LCLS. The surface doses to these materials at normal incidence in the center of the $8.275 \mathrm{KeV}$ LCLS FEL pulse at a position 15 meters downstream of the undulator exit are $0.318 \mathrm{eV} / a$ tom for aluminum, $0.802 \mathrm{eV} /$ atom for copper, and $7.28 \mathrm{eV} /$ atom for tantalum ${ }^{5}$. These doses are greater than the 0.01 to $0.1 \mathrm{eV} /$ atom range where damage is expected to occur.

We propose fabricating transmissive optics for the LCLS using alternating layers of Be and $\mathrm{B}_{4} \mathrm{C}$. Reflective multilayer optics have been successfully made using $\mathrm{Be}^{6}$ and $\mathrm{B}_{4} \mathrm{C}^{7}$. At $8.271 \mathrm{KeV}$, the optimal slice thickness for the $\mathrm{Be}$ and $\mathrm{B}_{4} \mathrm{C}$ system is $33 \mu \mathrm{m}$ which gives a $1 \pi$ phase shift between $x$ rays emerging from the $B e$ and $B_{4} C$ layers. The attenuation at $8.271 \mathrm{KeV}$ through the $33 \mu \mathrm{m}$ thick optic is negligible, the transmission through the $\mathrm{Be}$ is $99.5 \%$ while through the $\mathrm{B}_{4} \mathrm{C}$ the transmission is $98.2 \%$. More importantly the surface dose to the $\mathrm{Be}$ is $0.002 \mathrm{eV} / \mathrm{atom}$ and the surface dose to the $\mathrm{B}_{4} \mathrm{C}$ is $0.007 \mathrm{eV} / \mathrm{atom}$ at a position 15 meters downstream of the undulator exit at $8.271 \mathrm{KeV}$. Thus transmissive sputtered-sliced optics made of $\mathrm{Be}$ and $\mathrm{B}_{4} \mathrm{C}$ can take the full power of the $8.271 \mathrm{KeV}$ LCLS FEL pulse.

\section{Analytical formulation of time-slicing with off axis zone plates}

We derived a simple analytical model of the slice duration in terms of the zone plate and energy chirp parameters to evaluate the energy chirp scenarios and select zone plate parameters.

Suppose the zone plate was designed to have a focal length of $f_{0}$ at the nominal wavelength $\lambda_{0}$. It will have a central radius of $r_{1}=\sqrt{\lambda_{0} \cdot f_{0}}$ and its focal length at other wavelengths will be $f(\lambda)=r_{1}^{2} / \lambda$. A parallel pencil beam at the nominal wavelength striking the zone plate at a distance $X_{0}$ from its center will be focused to a point at an image distance $z_{\text {image }} \approx f_{0}$ downstream of the lens. If we place a screen in the plane perpendicular to the axis at the distance $z_{\text {image }}$ the focused beam will be displaced from the axis of the incident beam by the zone plate offset distance $X_{0}$. If we slightly change the wavelength, but leave the screen at the fixed distance $z_{\text {image }}$ the total displacement of the spot from the incident beam axis will be

$$
x(\lambda)=X_{0} \cdot\left(2-\frac{z_{\text {image }}(\lambda)}{z_{\text {image }}\left(\lambda_{0}\right)}\right) \approx X_{0} \cdot\left(2-\frac{f(\lambda)}{f_{0}}\right)=X_{0} \cdot\left(2-\frac{\lambda_{0}}{\lambda}\right) .
$$


If the duration of the full LCLS FEL pulse is $T$, and a chirp is introduced such that the wavelength changes from $\lambda_{0}(1-\Delta)$ in the beginning of the pulse to $\lambda_{0}(1+\Delta)$ at the end, and the pulse starts at time $t=0$, then the wavelength as a function of time is

$$
\lambda(t)=\lambda_{0} \cdot\left(1-\Delta+\Delta \cdot \frac{2 \cdot t}{T}\right) .
$$

Substituting the time dependent wavelength into the equation for the spot position, taking a time derivative and evaluating it at $t=T / 2$ (which is the time that the chirp reached the nominal wavelength $\lambda_{0}$ ) gives an equation for the speed of the focused spot near the nominal wavelength:

$$
\dot{x}=\frac{2 \cdot \Delta \cdot X_{0}}{T} .
$$

The duration of the time slice selected by a pinhole or slit at the position of the focused spot depends on the spot speed, the width of the slit, and the width of the focused spot. If $D$ is the smaller of the diameter of the incident beam or the aperture of the manufactured zone plate, the diffraction limited focal spot at a distance zimage from the optic has approximately a Gaussian shape with a FWHM of $\Gamma=a \cdot \frac{\lambda_{0}}{D} \cdot z_{\text {inage }}$ with the constant $a \approx 1.08$. In the limit of an infinitely thin slit, or if the size of the sample is much smaller than the focused spot, the FWHM of the time slice will be just the time needed for the spot to move a distance equal to its FWHM:

$$
\tau_{\text {slice }}=\frac{\Gamma}{\dot{x}}=\frac{a}{2} \cdot \frac{\lambda_{0} \cdot z_{\text {image }} \cdot T}{D \cdot \Delta \cdot X_{0}}
$$

As expected, the larger the zone plate offset, $X_{0}$, the shorter the time slice. Unfortunately larger zone plate offsets imply smaller zone plate feature sizes. Using the fact that the radius of the $n^{\text {th }}$ zone is $r_{n}=r_{1} \cdot \sqrt{n}$ and that the maximum radius from the zone plate optical axis to its outer radius is $X_{0}+1 / 2 \cdot D=r_{1} \cdot \sqrt{n_{\max }}$, we find that the offset as a function of the smallest zone width, $\delta=r_{1} \cdot\left(\sqrt{n_{\max }}-\sqrt{n_{\max }-1}\right)$, is given by:

$$
X_{0}=\frac{1}{2 \cdot \delta} \cdot\left(\lambda_{0} \cdot z_{\text {image }}+\delta^{2}-\delta \cdot D\right)
$$

Inserting this result into the equation for the FWHM of the time slice yields:

$$
\tau_{\text {slice }}=a \cdot \begin{gathered}
\lambda_{0} \cdot z_{\text {image }} \cdot T \cdot \delta \\
D \cdot \Delta \cdot\left(\lambda_{0} \cdot z_{\text {image }}+\delta^{2}-\delta \cdot D\right)
\end{gathered} .
$$

This equation gives the minimum duration (FWHM) of the time slice expected from a portion of an off-axis zone plate with minimum feature size $\delta$, and aperture $D$, as a function of zone plate image distance zimage, for given values of the pulse duration, $T$, and photon chirp half width, $\Delta$.

We now consider the time-slicing performance of a plausible sputtered-sliced off axis zone plate, one that has a minimum feature size of $\delta=0.33 \cdot \mu \mathrm{m}$ and a total aperture of 


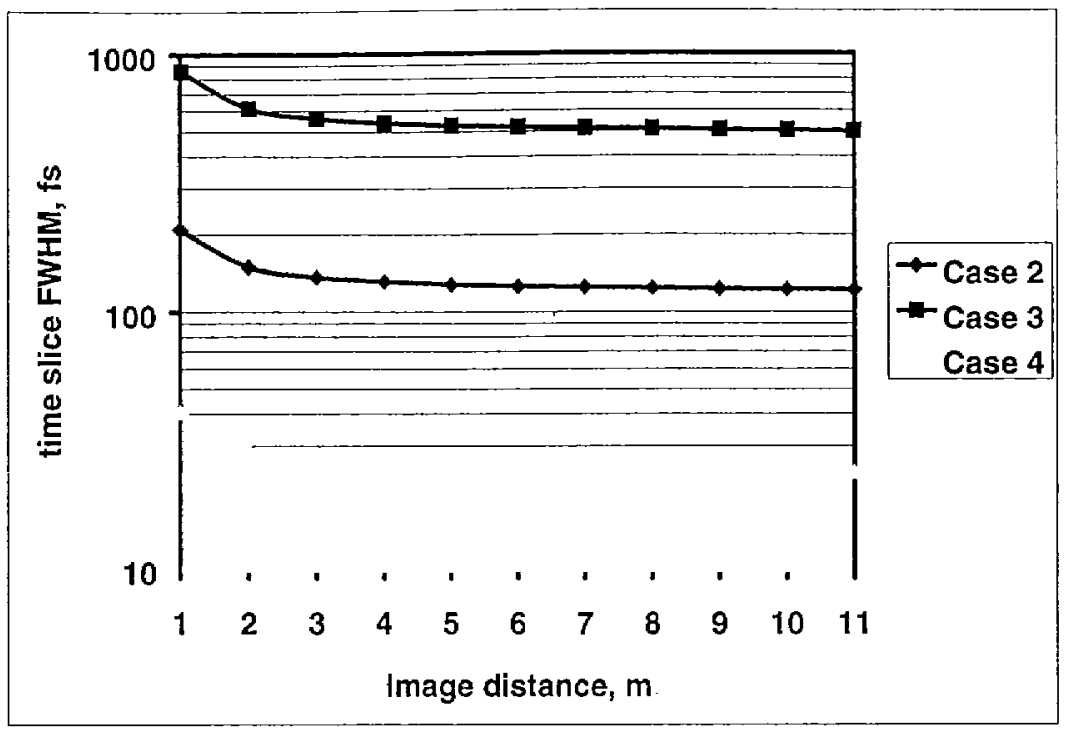

- Figure 3 - Slice times achievable with a zone plate having a minimum feature size of $0.35 \mu \mathrm{m}$, and an aperture of $200 \mu \mathrm{m}$ under the 3 energy chirp scenarios.

$D=200 \cdot \mu \mathrm{m}$. This lens has a fairly large aperture and small feature size, and although technically challenging, probably represents what is practical for the manufacture of a sputtered-sliced zone plate. Figure 3 shows a plot of the time slice (FWHM) vs. image distance for this off-axis zone plate for the 3 energy chirp scenarios and $a=1.08$. In general the slice time decreases with increasing image distance but approaches a limiting value for image distances over 4 meters. In cases 2 and 3 , the speed of the spot is so small that the spot barely moves a distance equal to its spot size over the full duration of the pulse. This lens will not produce a time-sliced FEL pulse under energy chirp scenarios 2 and 3 . Case 4, however, does produce a reduced pulse duration of just over 40 fs with a 1 meter image distance and asymptotically approaches a value of just above $20 \mathrm{fs}$. Although the slice duration gets smaller by several fs from 3 to 5 meters this must be traded against the decrease in the power density in the focal spot with increasing focal length.

\section{Off-axis zone plates for $\mathbf{5 0}$ fs time-slicing.}

As an alternative to evaluating the performance of a particular optic, we can use the formula to specify off-axis zone plate apertures and minimum feature sizes necessary to achieve a particular value for slice duration, say $50 \mathrm{fs}$, in each of the energy chirp scenarios. Solving the time slice equation for the minimum feature size yields

$$
\begin{aligned}
\delta= & \frac{1}{2} \cdot D+\frac{1}{2} \cdot \frac{a \cdot \lambda_{0} \cdot z_{\text {image }} \cdot T}{D \cdot \Delta \cdot \tau}- \\
& \frac{1}{2} \cdot \sqrt{D^{2}+2 \cdot \frac{a \cdot \lambda_{0} \cdot z_{\text {image }} \cdot T}{\Delta \cdot \tau}+\frac{a^{2} \cdot \lambda_{0}^{2} \cdot z_{\text {image }}^{2} \cdot T^{2}}{\tau^{2} \cdot D^{2} \cdot \Delta^{2}}-4 \cdot \lambda_{0} \cdot z_{\text {image }}}
\end{aligned} .
$$

Figure 4 shows plots of the minimum feature size, $\delta$, needed to achieve a $\tau=50$ is time-slice, as a function of image distance, $z_{\text {image }}$, for each of the energy chirp scenarios 

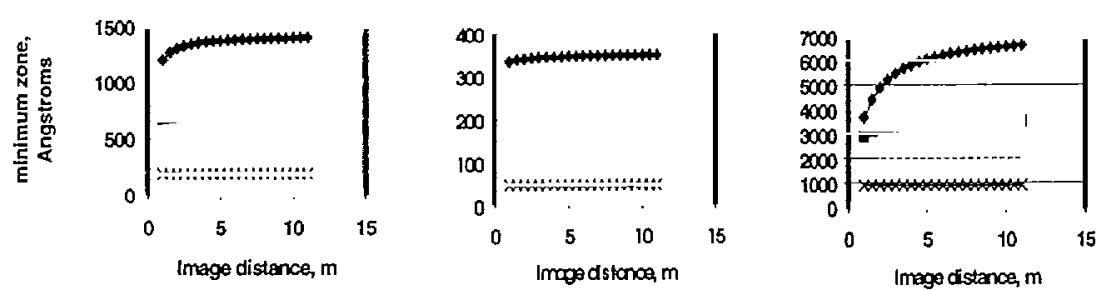

Lens aperture, $D=\left|\begin{array}{r}-200 \text { micron }-100 \text { micron } \\ 50 \text { micron } * 25 \text { micron }\end{array}\right|$

- Figure 4 - Minimum zone-plate feature sizes in $\AA$, needed to achieve a 50 is (FWHM) time-slice vs. image distance for the 3 energy chirp scenarios. The different colored lines represent lenses of differing aperture according to the key at the bottom of the figure.

and for different apertures, $D$. The four traces in each plot show the minimum feature sizes in $\AA$ for apertures of $200,100,50$, and $25 \mu \mathrm{m}$. The size of the largest aperture is limited by the FEL beam diameter. The larger apertures focus to smaller spot sizes and therefore require smaller spot speeds in order to satisfy the condition that the spot traverses its FWHM within $50 \mathrm{fs}$. The slower spot speeds required by the larger aperture lenses translates into larger minimum feature sizes. This is good from the standpoint of manufacturing sputtered-sliced lenses as larger zones are less effected by the growth defects that accumulate when sputtering very thick multilayers. The larger apertures also deliver more power density into the focused spot. Note that the number of zones required to achieve a given time-slice duration for a given energy chirp is a constant, independent of lens aperture, and serves as one measure of the difficulty in fabrication.

Case 2 , involving the $\pm 0.2 \%$ chirp over 130 is is shown on the left of figure 4 . This case requires approximately 1400 zones to achieve a 50 fs time-slice. The largest lens requires feature sizes of 1200 to $1400 \AA$ over its $200 \cdot \mu \mathrm{m}$ aperture. Depositing such a large and fine-featured multilayer is at or beyond the current state-of-the-art. It may be possible to make such a structure by bonding several smaller deposits together. The smaller aperture alternatives for case 2 are also very challenging. The $25 \cdot \mu \mathrm{m}$ lens requires zones of order $100 \AA$.

Case 3 in the middle of figure 4 , involving the $\pm 0.25 \%$ chirp over 670 fs, as expected, imposes even more fantastic, and probably unrealistic, requirements on the off-axis zone plate. This case requires around 5600 zones. Minimum feature sizes for the $200 \cdot \mu \mathrm{m}$ aperture are around $350 \AA$.

Case 4 on the right of figure 4 , involving the $\pm 2.0 \%$ chirp over $255 \mathrm{fs}$, presents very realistic requirements on the off-axis zone plate. This case requires approximately 300 zones. The largest aperture lens is seen to require a $200 \cdot \mu \mathrm{m}$ deposit with 4000 to 7000 A minimum features making it similar to sputtered-sliced optics previously reported. 


\section{Photon Flux Density Delivered to Sample}

One must also take into account the photon density at the sample when trading various time-slice schemes. In particular, the dependence of photon density on image distance is important since longer image distances translate into larger zones making fabrication easier. For structural studies of biomolecules a photon density of 200 photons / $\AA^{2}$ was mentioned as the threshold for damage. Presumably imagery at or below this density can be conveniently obtained at other facilities by integrating lower fluxes for longer times. Therefore it is of interest to see if the off-axes zone-plate time-slicing technique can be used at the LCLS to deliver $>200$ photons $/ \AA^{2}$ in $<50$ fs (FWHM), something that cannot be done at other facilitates.

The central photon density of the unfocused Gaussian LCLS FEL is given by:

$$
\rho_{\text {beam }}=\frac{P_{\text {sat }}}{2 \cdot \pi \cdot \sigma_{\text {beam }}^{2} \cdot E_{\text {photon }}} \cdot \tau_{\text {beam }} .
$$

At $E_{\text {photon }}=8.271 \cdot \mathrm{KeV}$ the FEL intensity distribution has $\sigma_{\text {beam }} \approx 37 \cdot \mu \mathrm{m}$ at a distance of 65 to 70 meters from the undulator center and a nominal saturated power level of $P_{s a t}=9.6 \cdot G$ watt according to the design study formalism. This works out to a central photon density of $\sim 200$ photons $/ \mathrm{nm}^{2}$ when integrated over the entire $\tau_{\text {beam }}=255 \cdot \mathrm{fs}$ pulse length. Thus the intensity of the raw FEL beam must be raised by at least a factor of 100 to achieve photon densities $>200$ photons $/ \AA^{2}$ even when using the full $255 \mathrm{fs}$ pulse.

A linear zone plate that focuses in 1 dimension will increase the central photon density by the ratio of the $\sigma$ of the unfocused beam to the $\sigma$ of the focused spot. If focusing is achieved in both dimensions, as with a circular zone plate or with crossed $X$ and $Y$ linear zone plates, the photon density will increase by this ratio squared. At the same time the photon density will be decreased by the efficiency of the lens $\varepsilon$, or by $\varepsilon^{2}$ for two lenses, as well as the loss of beam due to the finite aperture of the system. Using the equation for the diffraction limited spot size of a focusing optic having aperture $D$, the central photon density of the focused spot integrated over a slice time $\tau_{\text {slice }}$ will be:

$$
\begin{aligned}
\rho_{\text {slice }} & =\varepsilon^{n} \cdot\left(\frac{\sigma_{\text {beam }}}{\sigma_{\text {focus }}}\right)^{d} \cdot\left(\frac{1}{\sqrt{2 \cdot \pi} \cdot \sigma_{\text {beam }}} \cdot \int_{-D / 2}^{D / 2} e^{\left(-\frac{1 x^{2}}{2 \sigma_{\text {beam }}^{2}}\right)} \cdot d x\right)^{2} \cdot \rho_{\text {beam }} \cdot \frac{\tau_{\text {slice }}}{\tau_{\text {beam }}} \\
& =\frac{\varepsilon^{n} \cdot P_{\text {sat }}}{2 \cdot \pi \cdot E_{\text {photon }} \cdot \sigma_{\text {beam }}^{2}} \cdot\left(\frac{2 \cdot \sqrt{2 \cdot \ln (2) \cdot \sigma_{\text {beam }} \cdot D}}{a \cdot \lambda 0 \cdot z}\right)^{d} \times \\
\left.\left(\frac{1}{\sqrt{2 \cdot \pi} \cdot \sigma_{\text {beam }}} \cdot \int_{-D / 2}^{D / 2} e^{\left(-\frac{1}{2} x_{\text {beam }}^{2}\right.}\right) \cdot d x\right)^{2} \cdot \tau_{\text {slice }} &
\end{aligned}
$$

where $n$ is the number of lenses, $d$ is the number of focused dimensions and the integral is the aperture efficiency factor.

This function gives the cumulative photon density produced by a system of off-axes zone plates at a fixed point in space integrated over the duration of the time slice. The photon 
density is linearly proportional to the FEL saturated power level, $P_{s a t}$, and slice duration, $\tau_{\text {slice }}$. The value of the saturated FEL power level for each of the chirp scenarios is not known and will be difficult to predict due to its dependence on the election density in the undulator which is in turn extremely sensitive to the chirp induced enchantment of the coherent synchrotron emission ${ }^{\dagger}$. Nevertheless, in what follows, we assume that the FEL achieves its nominal saturated power during the chirp, which for the $8.275 \mathrm{KeV}$

fundamental is $P_{\text {sal }}=9.6$. Gwatts .

Under this assumption we consider 3 separate optical systems. The first is a single offaxis linear zone plate producing a line focus at the sample in 1 dimension. The second consists of two off-axis linear zone plates, one focusing in the $x$ direction followed by another focusing in the $y$ direction. This system produces a spot focus at the sample but its intensity is somewhat reduced by the efficiency of the second lens. The third optical system consists of a single, off-axis, circular zone plate producing a spot focus at the sample.
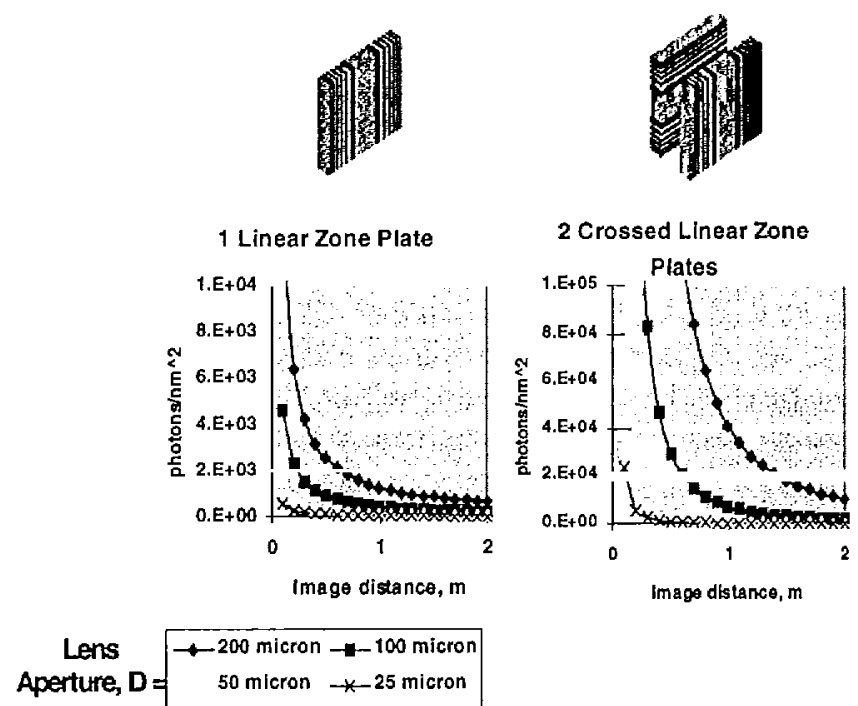

Aperture, $D=50$ micron $-*-25$ micron
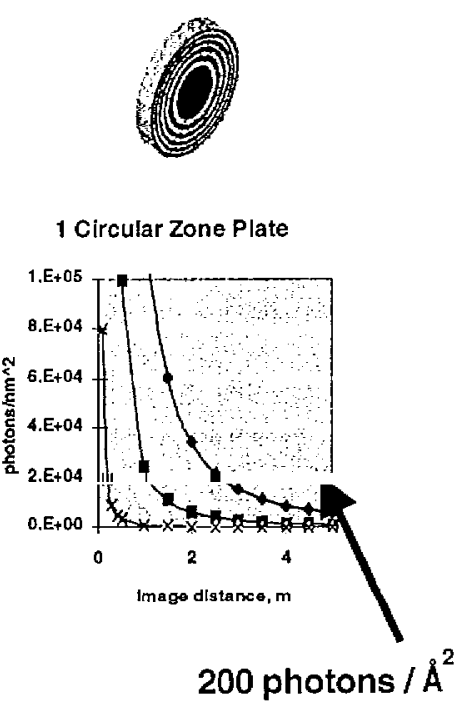

- Figure 5. Cumulative photon density at sample vs. image distance for 50 is time-slices produced by the zone-plate optical systems illustrated above each graph. The different data sets in each graph represent lenses of differing apertures listed in the key below the graphs. The two systems on the right are capable of producing photon densities greater than the 200 photon $\AA^{2}$ threshold for damage to biomolecules.

Figure 5 shows the resulting cumulative photon densities for the three optical systems as a function of image distance. The different curves in each plot are for lenses of deferring aperture ranging from $200 \mu \mathrm{m}$ down to $25 \mu \mathrm{m}$. The results for the single linear zone plate show that the photon densities are always below the interesting level of 20000 photons / $\mathrm{nm}^{2}$ for all apertures below $200 \mu \mathrm{m}$ having image distances $>0.1$ meters. The case of an optical system consistent of two crossed linear zone plates is more interesting. Two crossed linear zone plates are capable of producing photon densities $>20000$ photons / $\mathrm{nm}^{2}$ and at several different apertures. Systems of 2 crossed linear lenses having $200 \mu \mathrm{m}$

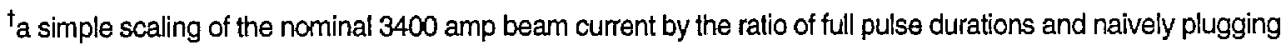
the resulting currents into the FEL power equations given in the design study yields saturated power levels of 31 Gwatts for case 2, 1.6 Gwatts for case 3, and the nominal 9.6 Gwatts for case 4. 
apertures and image distances $<1.46$ meters, or having $100 \mu \mathrm{m}$ apertures and image distances $<0.61$ meters, or having $50 \mu \mathrm{m}$ apertures and image distances $<0.19$ meters will all produce $50 \mathrm{fs}$ spots with cumulative photon densities $>20000$ photons $/ \mathrm{nm}^{2}$. Finally, as the figure shows, the optical system consisting of a single circular off-axis zone plate does the best, producing 50 fs time-sliced spots with photon densities above the 20000 photons $/ \mathrm{nm}^{2}$ threshold for image distances as long as 2.67 meters for a $200 \mu \mathrm{m}$ aperture, down to 0.3 meter focal lengths for $50 \mu \mathrm{m}$ apertures.

Figures 4 and 5 may be used to design 50 fs off-axis zone plate time-slicing optical systems as follows:

1) First, choose an optical system, from either 1 linear zone plate, 2 crossed linear zone plates, or 1 circular zone plate. For this example we pick the optical system consisting of a single circular zone plate.

2) Then choose an image distance and aperture giving the desired photon density using figure 5. For this example, in the graph for the circular optic, we choose an aperture of $200 \mu \mathrm{m}$ at an image distance of 2.67 meters to give a photon density of 20000 photons $/ \mathrm{nm}^{2}$ in a 50 fs slice.

3) Then identify the minimum feature size needed to give a 50 fs time-slice with the chosen image distance and aperture using figure 4. For this example, under chirp scenario 4, we find that a $200 \mu \mathrm{m}$ aperture with an image distance of $2.67 \mathrm{~m}$ requires a minimum feature size of $\sim 5300 \AA$.

4) Calculate the offset from the chosen minimum feature size, image distance, and aperture, using the offset equation. The required zone plate offset for this example is $277.5 \mu \mathrm{m}$.

5) Finally from the offset, image distance, and source distance, calculate the required focal length.

The resulting zone plate specifications for this example are given in table $Z$. The circular zone plate focal length is $2.546 \mathrm{~m}$, about $1.6 \%$ longer than the focal length calculated from the thin lens law due to the Gaussian beam shape. The zone plate pattern is fabricated by sputtering alternating layers of $\mathrm{Be}$ and $\mathrm{B}_{4} \mathrm{C}$ onto a wire core having a $177.5 \mu \mathrm{m}$ radius.

\begin{tabular}{|l|l|c|c|}
\hline Parameter & Value & Units \\
\hline & & \\
\hline & Object Distance & 53.99 & meters \\
\hline Image Distance & 2.67 & meters \\
\hline Focal length & 2.587 & meters \\
\hline Offset & 277.5 & microns \\
\hline & Oore radius & 177.5 & microns \\
\hline Inner zone width & 1.08 & microns \\
\hline Outer zone radius & 377.3 & microns \\
\hline & Outer zone width & 0.51 & microns \\
\hline
\end{tabular}

- Table 2 - Parameters of circular zone plate for 50 fs time-slicing 


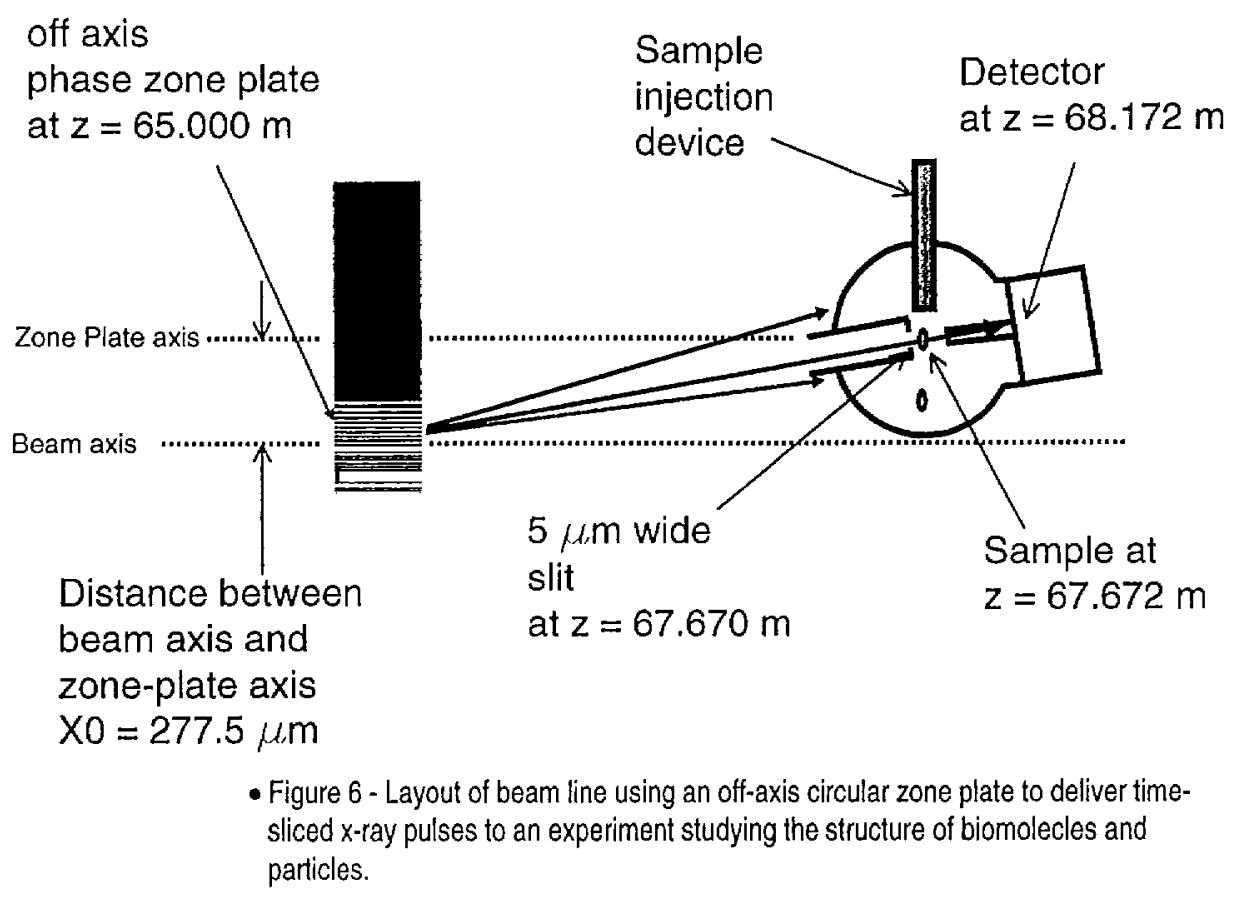

The deposit is $200 \mu \mathrm{m}$ thick extending to an outer radius of $377 \mu \mathrm{m}$ and contains 291 active zones. This zone plate is about $2 \times$ larger in diameter and active radius than previously fabricated sputtered-sliced circular zone plates but it also has fewer and larger zones so its fabrication is probably within the current state-of-the-art.

This analysis shows that practical sputtered-sliced off-axis zone plates could certainly be used to time-slice the LCLS FEL pulse to less than 50 fs in energy chirp case 4, and with some advance in the state-of-the-art, it may be feasible to fabricate off-axis zone plates for case 2. This invites further detailed analysis, in particular of the use of the specified sputtered-sliced circular zone plate designed above, to deliver time-sliced FEL pulses, under energy chirp scenario 4 , to a typical experimenter studying structural biology.

\section{Numerical simulation of off-axis zone zone plate time-sliced beam for delivered to user.}

We developed a numerical simulation based on the Gaussian properties of the FEL beam and Kirchhoff's diffraction theory to model the performance of the proposed circular zone plate when used to provide a focused, $8.27 \mathrm{KeV}$, time-sliced, $x$-ray pulse to a typical user who, for example, might be doing structural studies of biomolecules and particles.

Figure 6 shows the layout of the beam line and experimental apparatus. The zone plate is located at a distance $z=65 \cdot \mathrm{m}$ from the center of the undulator, with its optical axis offset by $277.5 \mu \mathrm{m}$ from the FEL beam axis. An apparatus for injecting biological samples into the focused spot is located a distance $2.67 \mathrm{~m}$ downstream of the zone plate and offset from the FEL beam by $277.5 \mu \mathrm{m}$. The injector fires biological particles a few $\mathrm{mm}$ behind an opaque wall located at the focus of the zone plate. The wall has a $5 \mu \mathrm{m}$ diameter pinhole located at the position of the focused spot at the center of the chip. The injector is synchronized to the FEL pulse so that the specimens arrive behind the pinhole as the focused spot sweeps by. The detector, located downstream, records the photons diffracted from the sample. 

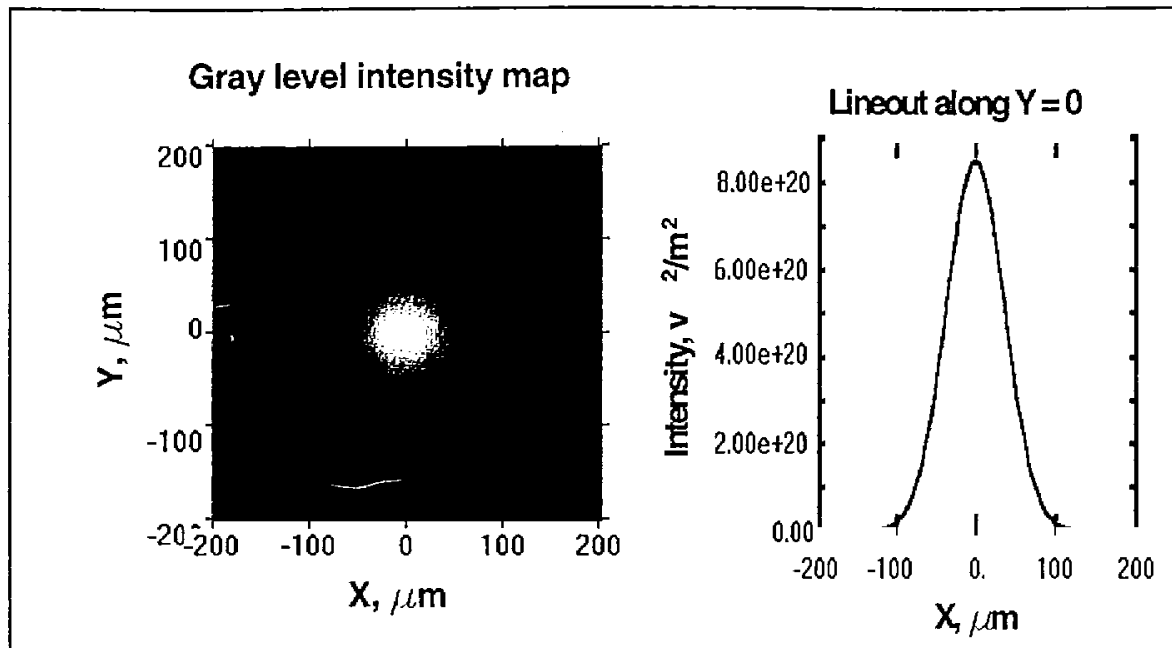

Figure 7 - Gray-level intensity map and lineout of the electric field intensity of the raw 8.275 KeV Gaussian beam just upstream of the off-axis zone plate.

To illustrate the use of the numerical simulation, we first use it to calculate the diffraction pattern produced by the off-axis zone plate at the central wavelength of the chirp, $\lambda_{0}=0.149854 \cdot \mathrm{nm}$ corresponding to a photon energy of $E_{\text {photon }}=8.275 \cdot \mathrm{KeV}$.

The simulation starts by calculating the electric field on a grid of points transverse to the beam axis and just upstream of the zone plate using the formula for the $8.275 \mathrm{KeV}$ Gaussian beam given in reference 5. Figure 7 shows a gray level map and line-out of the transverse electric field intensity upstream of the lens according to the Gaussian formula.

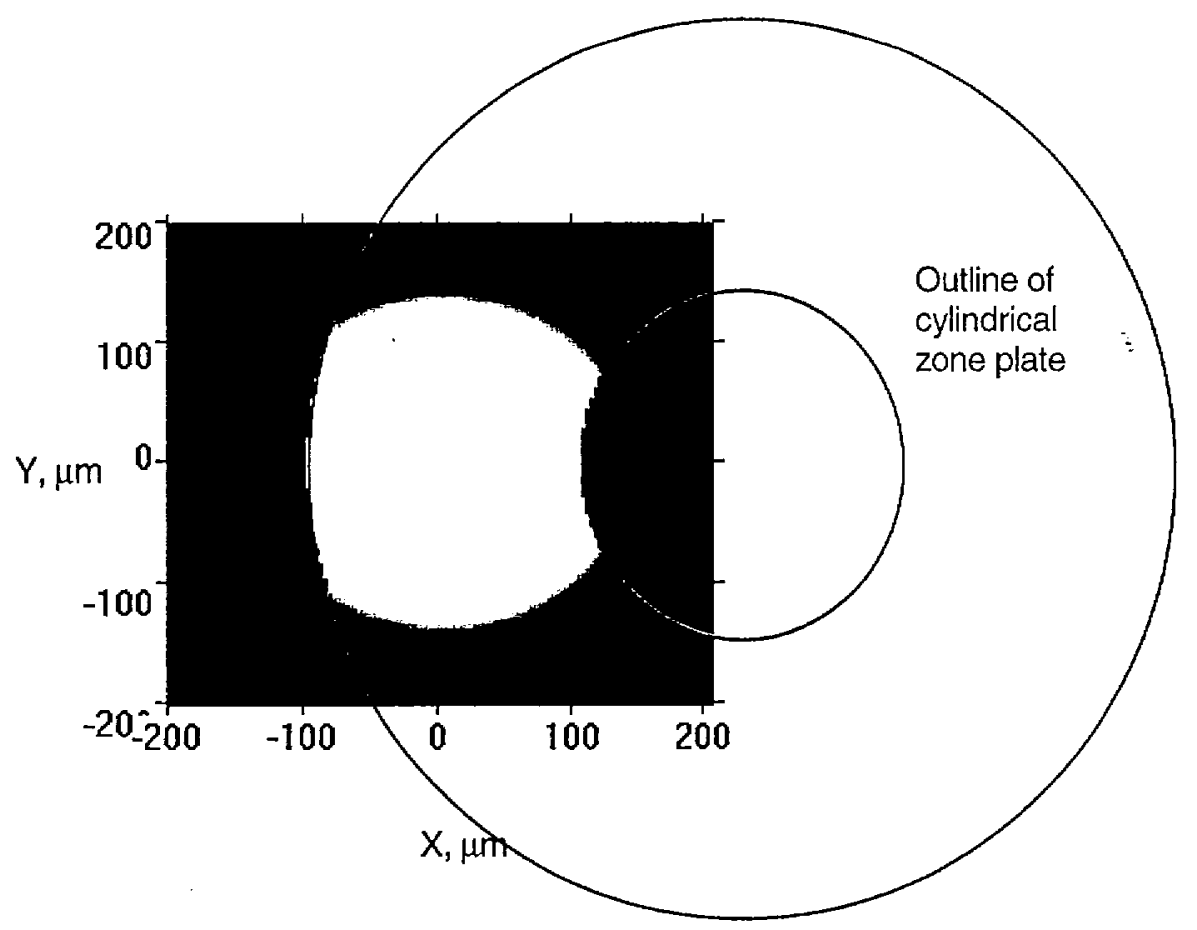

- Figure 8-Overexposed gray level map of the beam emerging from the lens. The active area of the lens changes only the phase of the electric field. 
The $8.275 \mathrm{KeV}$ raw FEL beam intensity distribution has a FWHM of $87 \mu \mathrm{m}$ and a peak electric field intensity of $8.47 \times 10^{20} \mathrm{volts}^{2} / \mathrm{m}^{2}$.

To simulate the action of the lens, the program multiplies the electric field at each point on the grid by the amplitude and phase shift experienced by $8.275 \mathrm{KeV}$ photons passing through the $33 \mu \mathrm{m}$ thickness of the material making up the zone in the lens at the grid point position. The modified field points represent the electric field just downstream of the optic. Figure 8 shows an overexposed gray level intensity distribution of this modified electric field overlaid with a drawing outlining the active area of the lens. The image was overexposed by altering the intensity to gray level map to show the active boundaries of the lens. Otherwise within the active area of the lens, the intensity of the portion of the beam exiting the lens is nearly identical to the incident beam intensity since the lens materials, $\mathrm{Be}$ and $\mathrm{B}_{4} \mathrm{C}$, are very transparent to $8.275 \mathrm{KeV}$ photons, and the induced phase shift is not discemible in the intensity distributions.

The program calculates the resulting electric field, $\Psi_{s}$, at a grid of points, $\left(x_{s}, y_{S}\right)$, transverse to the beam axis in the plane of the sample using the Kirchhoff diffraction integral:

$$
\begin{aligned}
& \Psi_{S}\left(x_{S}, y_{S}, z_{S}\right)=K_{f} \cdot e^{\frac{1}{2} \cdot \frac{i \cdot k \cdot\left(x_{S}^{2}+y_{S}^{2}\right)}{\Delta z}} \times \\
& \quad \times \iint\left\{\Psi_{M o d}\left(x_{L}, y_{L}, z_{L}\right) \cdot e^{\left.\frac{1}{2} \cdot \frac{i \cdot k \cdot\left(x_{L}^{2}+y_{L}^{2}\right)}{\Delta z}\right\}}\right\} e^{\frac{1}{2} \cdot \frac{i \cdot k \cdot x_{S} x_{L}}{\Delta z}} \cdot e^{\frac{1}{2} \cdot \frac{1 \cdot k \cdot y_{S} \cdot y_{L}}{\Delta z}} \cdot d x_{L} \cdot d y_{L}
\end{aligned}
$$

, where $\Delta z=z_{S}-z_{L}$ is the distance between the lens plane and the sample plane. The integral sums $\Psi_{\text {Mod }}$, the optic modulated electric field, times some exponential factors, over the traverse coordinates of the lens plane, $\left(x_{L}, y_{L}\right)$. The integral is recognized to be a 2-dimensional Fourier transform of the "shifted" field,

$$
\Psi_{M o d}\left(x_{L}, y_{L}, z_{L}\right) \cdot e^{\frac{1}{2} \cdot \frac{i \cdot k \cdot\left(x_{L}^{2}+y_{L}^{2}\right)}{\Delta z}} \text {, in the "frequencies" } \frac{k \cdot x_{S}}{\Delta z} \text { and } \frac{k \cdot y_{S}}{\Delta z} \text { and is rapidly }
$$

solved using a fast Fourier transform algorithm.

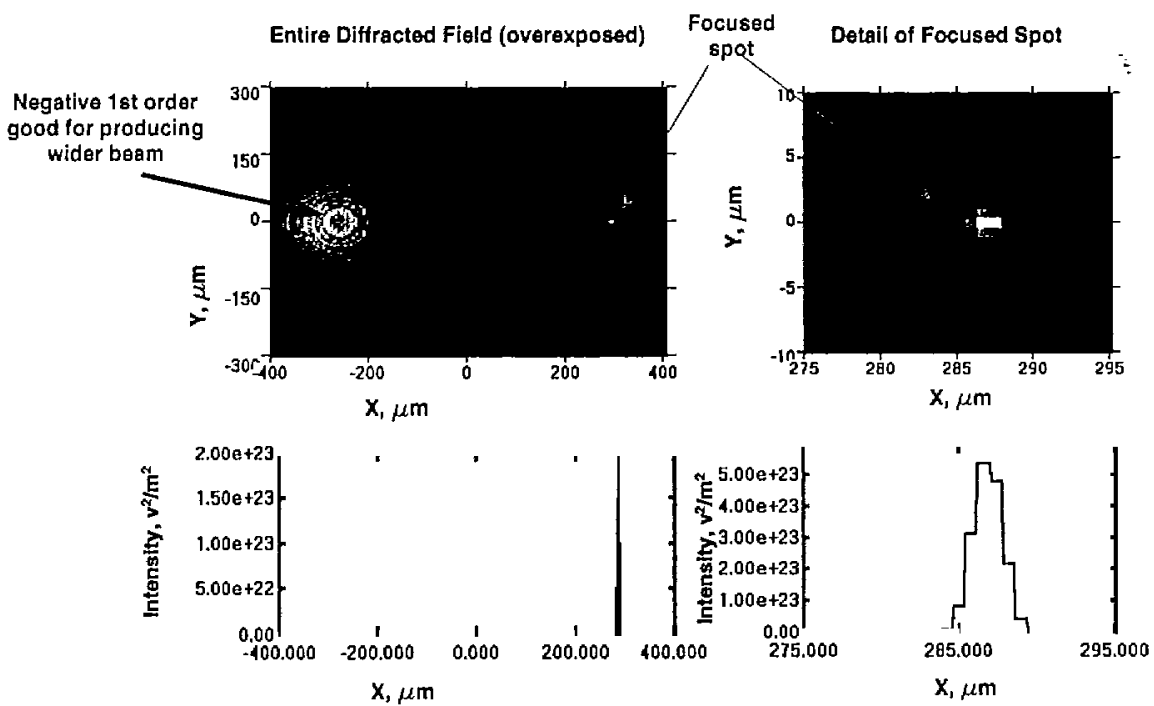

- Figure 9 - Calculated diffraction pattern in plane of sample produced by circular zone plate struck off-axis by $8.275 \mathrm{KeV}$ FEL. 
The resulting electric field intensity in the plane of the sample is shown in figure 9 . The overexposed image on the left spans an area of $800 \times 600 \mu \mathrm{m}$. A zone plate has both positive (converging) and negative (diverging) focusing orders of decreasing intensity. $A$ perfect phase zone plate displays only the odd orders. The first order positive focus is visible on the right side of the image, at the coordinates $(x, y) \approx(300 \cdot \mu \mathrm{m}, 0 \cdot \mu \mathrm{m})$ as a sharply focused spot. This is where the sample is located and contains over $30 \%$ of the beam power. On the left, near the position $(-300,0)$ is the divergent $-1^{\text {st }}$ order focus which also contains over $30 \%$ of the beam power. Although of no use here, the divergent $-1^{\text {st }}$ order focus is a fine source of coherent radiation in applications requiring larger beam diameters and / or beams of lower intensities. At the center of the image, surrounding the coordinates $(0,0)$ is the faint $0^{\text {th }}$ order beam which results from incomplete cancellation of the forward going light (because of absorption the $\mathrm{Be} / \mathrm{B}_{4} \mathrm{C}$ lens is not a perfect phase plate). Without overexposure the $-1^{\text {st }}$ order and the $0^{\text {th }}$ order would not be visible on a scale where the focused spot is unsaturated. As the intensity line-out at the bottom-left of figure 9 shows, the intensity of the $-1^{\text {st }}$ and $0^{\text {th }}$ order beams are insignificant compared to the intensity of the focused $1^{\text {st }}$ order spot.

On the right of Figure 9 is an enlargement of the $20 \times 20 \mu \mathrm{m}$ region containing the $+1^{\text {st }}$ order focus. The granularity of the FFT is apparent. As shown in the line-out the spot is nearly Gaussian with a peak intensity of $5.3 \times 10^{23}$ volt $^{2} / \mathrm{m}^{2}$, about $600 \times$ more intense than the unfocused beam. The FWHM is $2.2 \mu \mathrm{m}$, which agrees with the diffraction-limited prediction $\Gamma=a \cdot \frac{\lambda_{0}}{D} \cdot z_{\text {image }}$ when $a \approx 1.1$. The centroid of this spot is at the transverse coordinates $x=286.9 \cdot \mu m, y=0$, and since this simulation was done at the photon energy at the center of the chirp, the sample injector must be aligned so that the sample passes through this point.

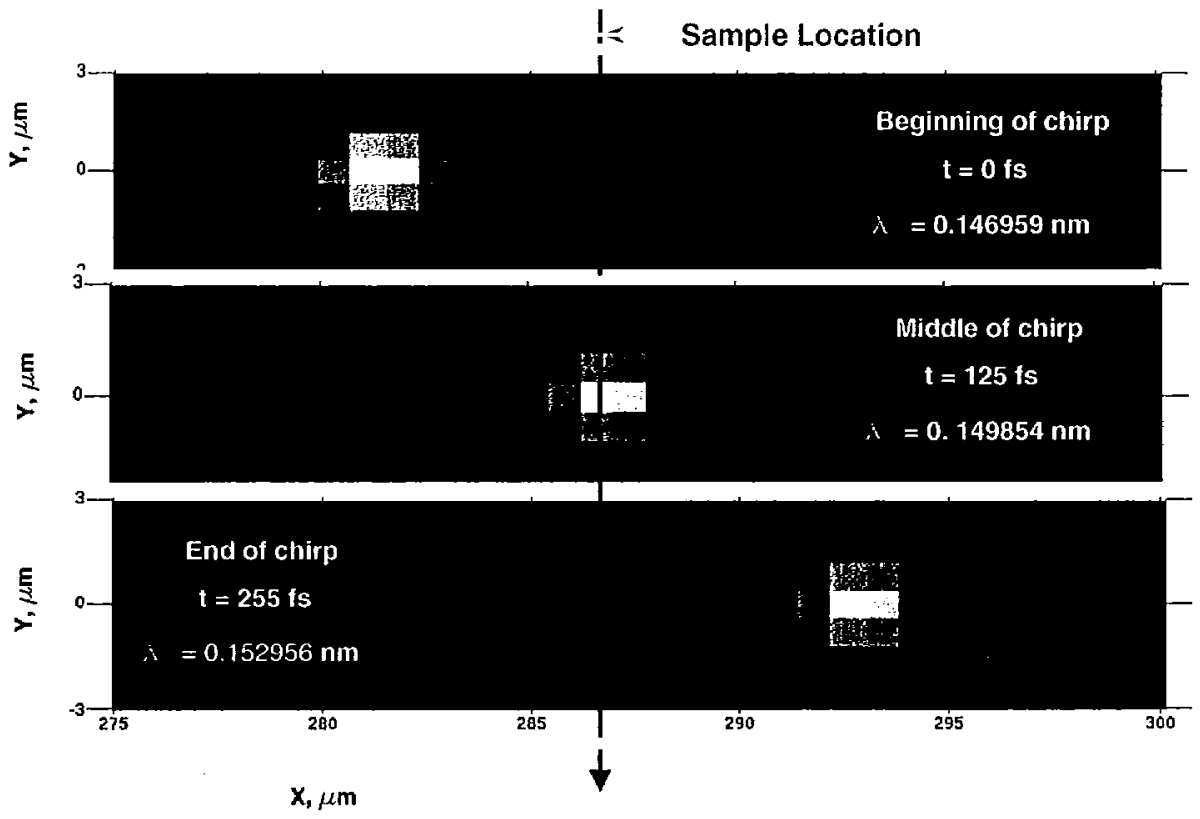

- Figure 10 - Focused spot at sample plane at beginning, middle, and end of chirp.

To simulate the chirped beam of case 4 , the program performs a loop over the pulse times from $t=0 \mathrm{fs}$ (beginning of pulse) to $t=255 \mathrm{fs}$ (end of pulse) in steps of $5 \mathrm{fs}$. For each time 
step the program calculates the FEL wavelength, power, and Gaussian beam parameters. It then evaluates the $\mathrm{Be} / \mathrm{B}_{4} \mathrm{C}$ optics constants for these wavelength photons and performs the FFT to calculate the electric field at the sample plane which it saves on the disk.

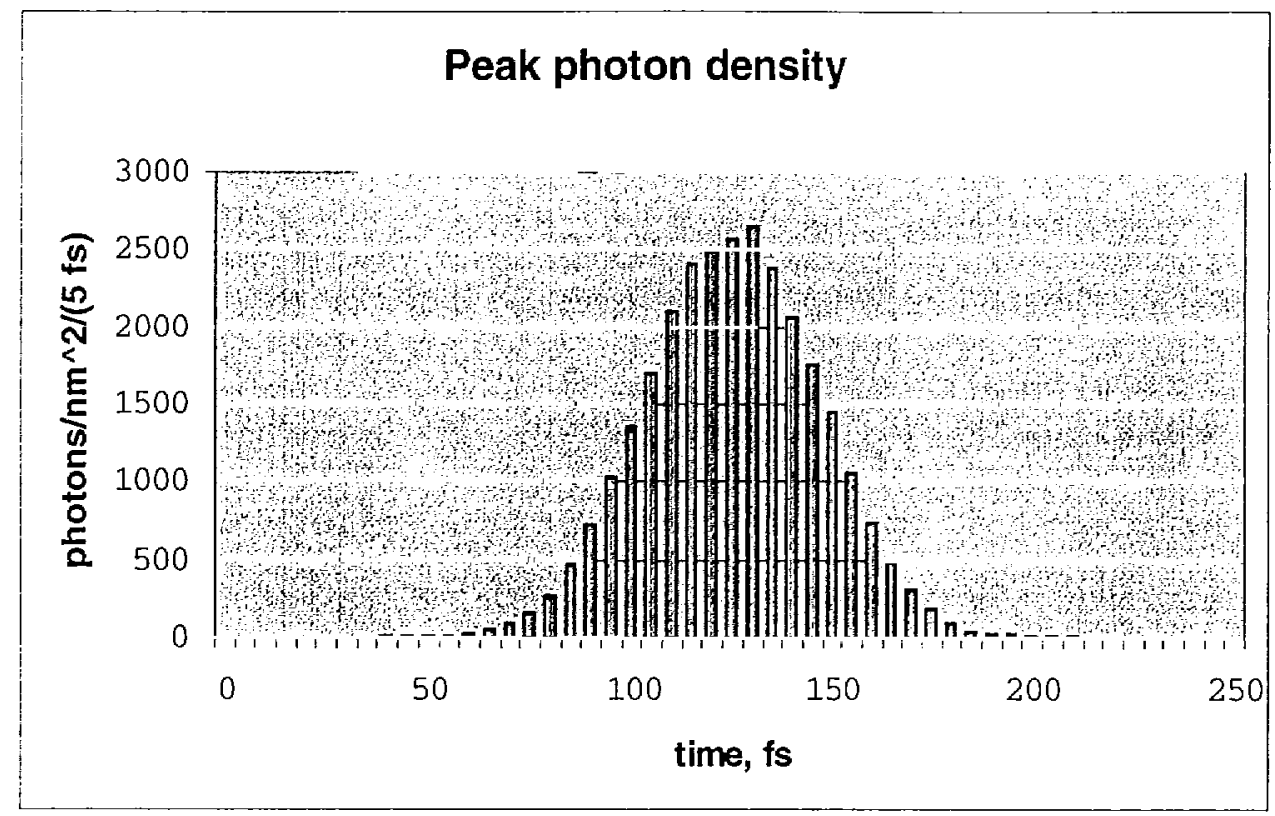

- Figure 11 - Photon density at the fixed location of the sample in 5 fs bins

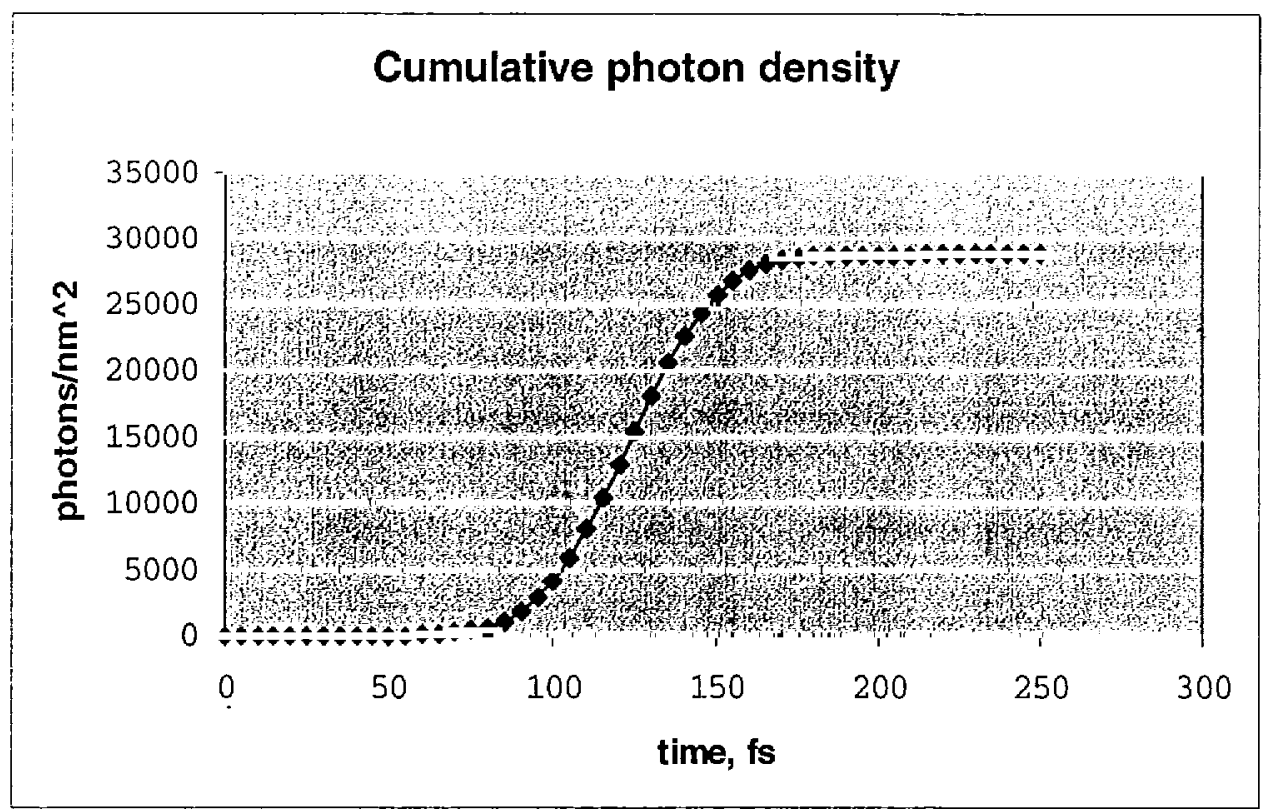

- Figure 12 - Cumulative photon density at the fixed sample position. 
Figure 10 shows the focused spots in the same area around the sample position for 3 of the 52 calculated diffraction patterns. The top image is of the $t=0$ is step, the middle is of the central $t=125$ fs step, and the bottom image is of the $t=255 \mathrm{fs}$ step. The 3 images clearly show the movements of the spot due to the wavelength change over the chirp and the change in spot size as the $z$ position of the focus moves from downstream to upstream of the sample plane. The line shows the position of the samples fired from the injector. The samples are much smaller, and move much slower than the focused spot so the time profile of the photon density seen by the sample is just given by the time history of the intensity at a fixed point in space as the focused spot sweeps across this position.

Figure 11 shows the time profile, in $5 \mathrm{fs}$ bins, of the photon density at the fixed point $(286.9,0)$ determined from the 52 calculated diffraction patterns. The pulse of $x$-rays that the sample sees has a FWHM of almost exactly $50 \mathrm{fs}$ in agreement with the analytical model. The peak photon density is just over 2500 photons $/ \mathrm{nm}^{2} / 5 \mathrm{fs}$.

Figure 12 shows the cumulative photon density at the same fixed point over the duration of the pulse. The cumulative photon density reaches 29000 photons $/ \mathrm{nm}^{2}$ or almost $1 / 3$ more than the 200 photon / A2 damage limit, close to the value predicted by the analytical model.

\section{Conclusions}

This study shows the performance expected from off-axis zone plate optical systems for delivering time-sliced, $8.275 \mathrm{KeV}$, LCLS FEL pulses to users under the 3 energy chirp scenarios. It is important to emphasize that these calculations assume that the LCLS achieves a saturated power level of 9.6 Gwatts during all phases of the chirp. The performance of off-axis zone-plate time-slicing systems is limited by the minimum feature size and maximum apertures capable of being produced by today's fabrication techniques. We find that the feature sizes and apertures necessary to provide $<50$ fs time-sliced FEL pulses with photon densities $>200 / \AA^{2}$ under energy chirp scenario 4 are similar to the feature sizes and apertures of sputtered-sliced zone-plates fabricated previously, and conclude that such systems are practical to build. But the fabrication technology would have to be considerably advanced beyond the-state-of-the-art to provide similar performance under energy chirp scenario 2 , and probably will never be capable of fabricating zone plates for energy chirp scenario 3.

\section{Acknowledgement}

This work was performed under the auspices of the U.S. Department of Energy by Lawrence Livermore National Laboratory under contracts No. W-7405-Eng-48 and DEAC03-76SF00515.

\section{References}

\footnotetext{
${ }^{1}$ Neutze, R. and Hajdu, J. (1997) "Femtosecond time resolution in X-ray diffraction experiments", Proc. Natt. Acad. Sci. U.S.A., 94, 5651-5655.

${ }^{2}$ Report of the LCLS SAC meeting, March 31, 2000.

${ }^{3}$ P. Emma, "Chirping the LCLS Electron Beam", LCLS Technical Note, LCLS-TN-00-6, (2000).

${ }^{4}$ R. M. Bionta, K. M. Skulina, J. Weinberg, "Hard x-ray sputtered-sliced phase zone plates", Appl. Phys. Lett. 64(8) p945. (1994).
} 
R. M. Bionta et. al., "Tabletop x-ray microscope using $8 \mathrm{KeV}$ zone plates", Optical Engineering.,29(6) p576, (1990).

K. Saitoh, K. Inagawa, K. Kohra, C. Hayashi, A. lida, and N. Kato, "Characterization of sliced multilayer zone plates for hard x rays", Rev. Sci. Instrum. 60 (7) p1519 (1989).

R. M. Bionta, A. F. Jankowski, and D. M. Makowiecki, "Fabrication and evaluation of transmissive multilayer optics for $8 \mathrm{KeV} x$ rays", Mat. Res. Symp. Proc., 103 p257 (1988).

R. M. Bionta et. al., "Sputtered-sliced multilayers: zone plates and transmission gratings for 8-KeV $x$ rays", SPIE 984 p247 (1988).

R. M. Bionta, "Transmission gratings that diffract $8 \mathrm{KeV}$ x rays", Appl. Phys. Lett. 51 (10) p725 (1987).

${ }^{5}$ R. M. Bionta, "Controlling dose to low Z solids at the LCLS", LLNL UCFL-ID-137222, (2000); LCLS-TN-00-3, (2000).

${ }^{6}$ K. M. Skulina, C. S. Afford, R. M. Bionta, D. M. Makowiecki, E. M. Gullikson, R. Soufli, J. N. Kortright, J. H. Underwood, "Molybdenum Be multilayer mirrors for nomal incidence in the extreme ultraviolet", Applied Optics, 34 (19) p3727 (1995).

${ }^{7}$ A. F. Jankowski, D. M. Makowiecki, "W/B 4 C multilayer x-ray mirrors", Optical Engineering, 30 (12) p2003 (1991). 